\title{
Magnetization Performance of LDPC Reduced-Complexity Decoding Algorithms
}

\author{
Manel Abdelhedi ${ }^{1}$, Omessaad Hamdi ${ }^{2}$, Ammar Bouallegue ${ }^{1}$ \\ ${ }^{1}$ SYSCOM Laboratory, ENIT, Tunis, Tunisia \\ ${ }^{2}$ LaBRI Laboratory, University of Bordeaux, Bordeaux, France \\ E-mail: abdelhedi_manel@yahoo.fr, hamdi@univ-tln.fr, ammar.bouallegue@enit.rnu.tn \\ Received March 20, 2010; revised April 21, 2010; accepted May 26, 2010
}

\begin{abstract}
Low-density parity-check (LDPC) codes are very efficient for communicating reliably through a noisy channel. N.Sourlas [1] showed that LDPC codes, which revolutionize the codes domain and used in many communications standards, can be mapped onto an Ising spin systems. Besides, it has been shown that the Belief-Propagation (BP) algorithm, the LDPC codes decoding algorithm, is equivalent to the ThoulessAnderson-Palmer (TAP) approach [2]. Unfortunately, no study has been made for the other decoding algorithms. In this paper, we develop the Log-Likelihood Ratios-Belief Propagation (LLR-BP) algorithm and its simplifications the BP-Based algorithm and the $\lambda$-min algorithm with the TAP approach. We present the performance of these decoding algorithms using statistical physics argument i.e., we present the performance as function of the magnetization.
\end{abstract}

Keywords: LDPC Codes, Ising Spin, LLR-BP Algorithm, BP-Based Algorithm, $\lambda$-Min Algorithm, TAP Approach

\section{Introduction}

Low-density parity-check (LDPC) codes were first discovered by Gallager [3], in his thesis, in 1962 and have recently been rediscovered by Mackay and Neal $[3,4]$. These codes can get very close to the Shannon limit by mean of an iterative decoding algorithm called BeliefPropagation (BP) algorithm [5].

This performance combined with their relatively simple decoding algorithm makes these codes very attractive for the next generation of digital transmission systems. Indeed, these codes have been chosen as a standard for the second Satellite Digital Video Broadcasting normalization (DVB-S2). So, the search for efficient implementations of decoding algorithms is being a challenge.

The implementation of the BP algorithm is difficult. That's why different simplifications of this algorithm were discovered. The BP algorithm can be simplified using the BP-Based algorithm [6] and the $\lambda$-min algorithm [7].

N. Sourlas [1] has shown in 1989 that there is a mathematical equivalence of error-correcting codes to some theoretical spin-glass models. This analogy has contributed to the present proximity of the statistical physics and the information theory. Therefore, the methods of statistical physics developed in the study of disordered systems proved to be efficient for studying the properties of these codes. One of these methods is the Thouless-AndersonPalmer (TAP) [2] approach which is shown equivalent to the BP algorithm by kabashima, et al. [8]. Unfortunately, no study has been made for the other decoding algorithms.

In this paper, we develop the Log-Likelihood RatiosBelief Propagation (LLR-BP), the BP-Based and the $\lambda$-min decoding algorithms with the TAP approach. Their performance is evaluated as a function of a statistical physics parameter which is the magnetization.

This paper is organized as follows. Section 2 corresponds to a general description of the LDPC codes and their decoding algorithm. Section 3 describes the similarity between the BP algorithm and the TAP approach. In Section 4, we develop the LLR-BP algorithm and its simplifications the BP-Based algorithm and the $\lambda$-min algorithm with the TAP approach. Finally and before concluding, simulation results as function of the magnetization are presented in Section 5.

\section{LDPC Codes and Decoding Algorithm}

\subsection{Low-Density Parity Check Codes}

Binary LDPC codes, are linear block codes defined by a 
sparse parity check matrix $A(M \times N)$, where $N$ denotes the codeword length and $M$ the number of paritycheck equations.

Using a notation similar to that in $[4,6]$, let $L(\mu)=$ $\left\{j / A_{\mu j}=1\right\}$ denote the set of bit $j$ that participate in check $\mu$. Similarly, we define the set of checks in which bit $j$ participates as $M(j)=\left\{\mu / A_{\mu j}=1\right\}$. We denote a set $L(\mu)$ with bit $j$ excluded by $L(\mu) \backslash j$, and a set $M(j)$ with parity check $\mu$ excluded by $M(j) \backslash \mu$. Finally, $s=\left(s_{1}, \ldots, s_{N}\right)$ denotes the transmitted codeword.

\subsection{Belief Propagation Algorithm}

This section summarizes according to [4,5], the iterative decoding of LDPC codes based on the BP algorithm. The likelihood of $s$ is given by $\prod_{j} f_{j}^{a}$, with $f_{j}^{a}=P\left(s_{j}=a\right)$.

Let $q_{\mu j}^{a}$ denotes the probability that bit $j$ of $s$ is $a$, given the information obtained via checks other than check $\mu$. The quantity $r_{\mu j}^{a}$ is meant to be the probability of check $\mu$ is satisfied if bit $j$ of $s$ is considered fixed at $a$ and the other bits have a separable distribution given by the probabilities $\left\{q_{\mu l}: l \in L(\mu) \backslash j\right\} \quad[6]$.

The standard iterative decoding algorithm based on the BP approach consists on the following main steps.

- Initialization: The variables $q_{\mu j}^{0}$ and $q_{\mu j}^{1}$ are initialized to the values $f_{j}^{0}$ and $f_{j}^{1}$ respectively.

\section{- Iterative processing:}

1) Define $\delta q_{\mu j}=q_{\mu j}^{0}-q_{\mu j}^{1}$ and for each $\mu$, $j \in L(\mu)$, and for $a=\{0,1\}$, compute

$$
\begin{aligned}
\delta r_{\mu j} & =\prod_{l \in L(\mu) \backslash j} \delta q_{\mu l} \\
r_{\mu j}^{a} & =\frac{1}{2}\left(1+(-1)^{a} \delta r_{\mu j}\right)
\end{aligned}
$$

2) For each $j$ and $\mu \in M(j)$, and for $a=\{0,1\}$ update

$$
\begin{gathered}
q_{\mu j}^{a}=\alpha_{\mu j} f_{j}^{a} \prod_{v \in M(j) \backslash \mu} r_{v j}^{a} \\
q_{j}^{a}=\alpha_{j} f_{j}^{a} \prod_{\mu \in M(j)} r_{\mu j}^{a}
\end{gathered}
$$

where $\alpha_{\mu j}$ and $\alpha_{j}$ are chosen such that

$$
q_{\mu j}^{0}+q_{\mu j}^{1}=1 \text { and } q_{j}^{0}+q_{j}^{1}=1 .
$$

3) Create the word $\hat{s}=\left(\hat{s}_{1} \ldots \hat{s}_{N}\right)$ the detection of the transmitted codeword such that:

$$
\begin{array}{ll}
\hat{s}_{j}=0 & \text { if } \delta q_{j}>0 \\
\hat{s}_{j}=1 & \text { if } \delta q_{j} \leq 0
\end{array}
$$

If $H . \hat{s}^{T}=0$ the decoding process ends. Otherwise, we pass to another iteration of BP. A failure is declared and the decoding process ends if the number of iterations exceeds the maximum number of iterations and $\hat{s}$ is not a valid codeword.

\section{Decoding Problem from Statistical Physics Point of View}

\subsection{Statistical Physics Analogy}

In the previous section, we have described the LDPC codes using the additive Boolean group $(\{0,1\},+)$. However, in order to apply methods of statistical physics, it is convenient to introduce an equivalent group, the multiplicative binary group $(\{+1,-1\}, \times)[1]$.

From a statistical physics point of view, the code can be regarded as a spin system. Each bit $S_{j}=(-1)^{s_{j}}$ is called a spin and takes values in $\{ \pm 1\}$ and the word $S=\left((-1)^{s_{1}}, \ldots,(-1)^{s_{N}}\right)$ can be seen as a collection of $N$ spins, called a configuration. The parity-check matrix gives rise to the interactions between the spins [1].

The decoding problem depends on posteriori like $P(S \backslash J)$ where $J$ is the evidence (received message or syndrome vector). By applying Bayes' theorem this posteriori can be written in the form [1].

$$
\begin{aligned}
P(S \backslash J) & =\frac{P(J \backslash S) P(S)}{\sum_{S} P(J \backslash S) P(S)} \\
& =\frac{1}{Z} \exp [\ln P(J \backslash S)+\ln P(S)]
\end{aligned}
$$

In the statistical physics description, the probability (1) can be expressed as a Boltzmann distribution at the inverse temperature $\beta$ [9].

$$
P(S \backslash J)=\frac{1}{Z} \exp [-\beta H(S \backslash J)]
$$

where $H(S \backslash J)$ is the Hamiltonian of the system.

In this Hamiltonian, we identify two components that are necessary for the analysis of LDPC codes.

- A term that guarantees that all parity checks are satisfied. It can be written with the Kronecker's delta $\delta[1]$.

$$
P(J \backslash S)=\prod_{\mu=1}^{M} \delta\left[J_{\mu}, \prod_{j \in L(\mu)} S_{j}\right]
$$

According [9], the $\delta$ 's can be replaced by a soft constraint.

$$
P(J \backslash S)=\text { const } \exp \left[\beta \sum_{\mu=1}^{M} J_{\mu} \prod_{j \in L(\mu)} S_{j}\right]
$$


where $\beta \rightarrow \infty$.

- A prior term that provides some statistical information on the dynamical variables $S$. It can be represented by the prior distribution

$$
P(S)=\frac{\exp \left(F \sum_{j=1}^{N} S_{j}\right)}{(2 \cosh F)^{N}}
$$

where $F$ is the external field. It is determined by the flip rate $f$ of channel noise as $F=\frac{1}{2} \ln \frac{1-f}{f}$.

So, the Hamiltonian $H(S \backslash J)$ is written as follows:

$$
H(S \backslash J)=-\sum_{\mu=1}^{M} J_{\mu} \prod_{j \in L(\mu)} S_{j}-\frac{F}{\beta} \sum_{j=1}^{N} S_{j}
$$

where $J_{\mu}$ is the muti-spin coupling.

\subsection{Decoding in the Statistical Physics}

The decoding process corresponds to finding local magnetization at the inverse temperature $\beta$, $m_{j}=\left\langle S_{j}\right\rangle_{\beta}$ and calculating estimates as [1].

$$
\hat{S}_{j}=\operatorname{sign}\left(m_{j}\right)
$$

The decoding performance in the statistical physics approach can be measured by the magnetization [1] defined by the overlap between the actual message and estimate:

$$
m=\left\langle\frac{1}{N} \sum_{j=1}^{N} S_{j} \hat{S}_{j}\right\rangle
$$

Here the overage $\langle\ldots\rangle$ is performed over the matrices $A$. This value provides information about the typical performance of the code.

The Magnetization is an order parameter which has a standard of judgment whether the whole system exhibits an ordered state or not.

- If $m=1$ the system is in an ordered phase called ferromagnetic phase.

- If $m=0$ the system is in a disordered phase called paramagnetic phase.

Two main methods can be employed for calculating the value of the magnetization: the replica method for diluted systems [9] and the TAP approach [8]. In this paper, we are interested only on the TAP approach.

\subsection{TAP Approach}

Kabashima, et al. [8] have shown the similarity between equations derived from the TAP [2] approach and those obtained from BP. The fields $q_{\mu j}^{a}$ correspond to the mean influence of sites other than the site $j$ and the fields $r_{\mu j}^{a}$ represent the influence of $j$ back over the system (reaction fields) [10].

The similarity can be exposed by observing that the likelihood $P\left(J_{\mu} \backslash S\right)$ is proportional to the Boltzmann weight [11]

$$
\omega_{B}\left(J_{\mu} \backslash\left\{S_{j}: j \in L(\mu)\right\}\right)=\exp \left(-\beta J_{\mu} \prod_{j \in L(\mu)} S_{j}\right)
$$

The conditional probability $r_{\mu j}^{S_{j}}$ can be seen as a normalized effective Boltzmann weight (effective Boltzmann probability) obtained by fixing the bit $S_{j}$ [10]

$$
\begin{aligned}
r_{\mu j}^{S_{j}} & =\alpha_{\mu j} \omega_{e f f f}\left(J_{\mu} \backslash S_{j}\right) \\
& =\alpha_{\mu j} \sum_{S_{l} l \in L(\mu) \backslash j} \omega_{B}\left(J_{\mu} \backslash\left\{S_{l}: l \in L(\mu)\right\}\right) \prod_{l \in L(\mu) \backslash j} q_{\mu l}^{\left(S_{l}\right)}
\end{aligned}
$$

Since spin variable $S_{j}$ takes only two values \pm 1 , it is convenient to express the BP/TAP algorithm using spin averages $\sum_{S_{j}= \pm 1} S_{j} q_{\mu j}^{S_{j}}$ and $\sum_{S_{j}= \pm 1} S_{j} r_{\mu j}^{S_{j}}$ rather than the distributions $q_{\mu j}^{S_{j}}$ and $r_{\mu j}^{S_{j}}$ themselves. We use $\delta q_{\mu j}=m_{\mu_{j}}$ to denote $\sum_{S_{j}= \pm 1} S_{j} q_{\mu j}^{S_{j}}$. Similar notation $\delta r_{\mu j}=\hat{m}_{\mu_{j}}$ is used for $\sum_{S_{j}= \pm 1} S_{j} r_{\mu j}^{S_{j}}$. Additionally, it was shown in [10] that the following statements hold

$$
\begin{gathered}
\hat{m}_{\mu j}=\tanh \left(\beta J_{\mu}\right) \prod_{l \in L(\mu)\rangle_{j}} m_{\mu l} \\
m_{\mu j}=\tanh \left(\sum_{v \in M(j) \backslash \mu} \tanh ^{-1}\left(\hat{m}_{v j}\right)+\beta F\right)
\end{gathered}
$$

The pseudo-posteriori can then be calculated

$$
m_{j}=\tanh \left(\sum_{\mu \in M(l)} \tanh ^{-1}\left(\hat{m}_{\mu j}\right)+\beta F\right)
$$

This provides a way for computing the Bayes optimal decoding as follows:

$$
\hat{S}_{j}=\operatorname{sign}\left(\delta q_{j}\right)=\operatorname{sign}\left(m_{j}\right)
$$

The result that $\delta q_{j}=m_{j}$ is demonstrated as follows:

$$
\begin{aligned}
m_{j} & =\left\langle S_{j}\right\rangle_{\beta} \\
& =\frac{1}{Z} \sum_{S} S_{j} \exp [-\beta H(S \backslash J)] \\
& =\sum_{S} S_{j} P(S \backslash J) \\
& =\sum_{S_{j}} S_{j} \sum_{S \backslash S_{j}} P(S \backslash J) \\
& =\sum_{S_{j}} S_{j} P\left(S_{j} \backslash J\right) \\
& =\delta q_{j}
\end{aligned}
$$




\section{LLR-BP Algorithm and its Simplifications with TAP Approach}

\subsection{LLR-BP Algorithm with TAP Approach}

In the LLR-BP algorithm instead of handling probabilities as in $[3,5]$ we are going to deal with the LLRs. In practice, using LLRs as messages offers implementation advantages over using probabilities or likelihood ratios because multiplications are replaced by additions and the normalization step is eliminated [11].

In this section, we try to develop the LLR-BP with the TAP approach. Let $x_{\mu j}$ and $y_{\mu j}$ be the LLR of bit $j$ which are sent from bit node $j$ to check node $\mu$ and from the check node $\mu$ to bit node $j$, respectively. From the statistical physics definition, the LLR is defined as follows [12]:

$$
x_{\mu j}=\frac{1}{2 \beta} \ln \frac{q_{\mu j}^{+1}}{q_{\mu j}^{-1}}=\frac{x_{\mu j}^{\prime}}{2 \beta}
$$

And

$$
y_{\mu j}=\frac{1}{2 \beta} \ln \frac{r_{\mu j}^{+1}}{r_{\mu j}^{-1}}=\frac{y_{\mu j}^{\prime}}{2 \beta}
$$

We need also the following results

$$
\begin{aligned}
\tanh \left(\beta x_{\mu j}\right) & =\tanh \left(\frac{1}{2} \ln \left(\frac{q_{\mu j}^{+1}}{q_{\mu j}^{-1}}\right)\right) \\
& =\frac{q_{\mu j}^{+1}-q_{\mu j}^{-1}}{q_{\mu j}^{+1}+q_{\mu j}^{-1}} \\
& =q_{\mu j}^{+1}-q_{\mu j}^{-1} \\
& =m_{\mu j}
\end{aligned}
$$

Like in (12), the variable $\hat{m}_{\mu j}$ can be also written as:

$$
\hat{m}_{\mu j}=\tanh \left(\beta y_{\mu j}\right)
$$

So, from Equations (5), (12) and (13) we have

$$
\begin{aligned}
x_{\mu j} & =\frac{1}{\beta} \tanh ^{-1} \tanh \left(\sum_{v \in M(j) \backslash \mu} \tanh ^{-1}\left(\hat{m}_{v j}\right)+\beta F\right) \\
& =\sum_{v \in M(j) \backslash \mu} y_{v j}+F
\end{aligned}
$$

Also, $y_{\mu j}$ can be written as

$$
\begin{aligned}
y_{\mu j} & =\frac{1}{\beta} \tanh ^{-1}\left(\hat{m}_{\mu j}\right) \\
& =\frac{1}{\beta} \tanh ^{-1}\left(\tanh \left(\beta J_{\mu}\right) \prod_{l \in L(\mu) \backslash j} \tanh \left(\beta x_{\mu l}\right)\right)
\end{aligned}
$$

Finally, the posteriori LLR of bit $S_{j}$ is

$$
x_{j}=\sum_{\mu \in M(j)} y_{\mu j}+F
$$

It's not easy to implement (15) which has the form of a product. Our idea is to decompose the check node update into sign and magnitude processing like in [13]. We have from (15)

$$
\tanh \left(\beta y_{\mu j}\right)=\tanh \left(\beta J_{\mu}\right) \prod_{l \in L(\mu)_{j}} \tanh \left(\beta x_{\mu l}\right)
$$

Replacing $\left(\beta J_{\mu}\right)$ by $\operatorname{sign}\left(\beta J_{\mu}\right) \times\left|\beta J_{\mu}\right|$ and $\left(\beta x_{\mu l}\right)$ by $\operatorname{sign}\left(\beta x_{\mu l}\right) \times\left|\beta x_{\mu l}\right|$ in (17) yields

$$
\begin{aligned}
\operatorname{sign}\left(\beta y_{\mu j}\right) & =\prod_{l \in L(\mu) \backslash j} \operatorname{sign}\left(\beta^{2} J_{\mu} x_{\mu l}\right) \\
& =\prod_{l \in L(\mu) \backslash j} \operatorname{sign}\left(J_{\mu} x_{\mu l}\right) \\
\tanh \left|\beta y_{\mu j}\right| & =\tanh \left|\beta J_{\mu}\right| \prod_{l \in L(\mu) \backslash j} \tanh \left|\beta x_{\mu l}\right| \\
\tanh \left|\frac{y_{\mu j}^{\prime}}{2}\right|= & \tanh \left|2 \frac{\beta J_{\mu}}{2}\right| \prod_{l \in L(\mu) \backslash j} \tanh \left|\frac{x_{\mu l}^{\prime}}{2}\right|
\end{aligned}
$$

Let $f(t)$ be defined by

$$
f(t)=-\ln \left(\tanh \left(\frac{t}{2}\right)\right)=\ln \frac{e^{t}+1}{e^{t}-1}
$$

Then, taking the logarithm of the inverse of both sides of (19) yields

$$
\begin{aligned}
& -\ln \tanh \left|\frac{y_{\mu j}^{\prime}}{2}\right|=-\ln \tanh \left|2 \frac{\beta J_{\mu}}{2}\right|+\sum_{l \in L(\mu) \backslash j}-\ln \tanh \left|\frac{x_{\mu l}^{\prime}}{2}\right| \\
& f\left(\left|y_{\mu j}^{\prime}\right|\right)=f\left(\left|2 \beta J_{\mu}\right|\right)+\sum_{l \in L(\mu) \backslash j} f\left(\left|x_{\mu l}^{\prime}\right|\right)
\end{aligned}
$$

The Equation (20) verifies

$$
f(f(t))=\ln \frac{\left(\frac{e^{t}+1}{e^{t}-1}\right)+1}{\left(\frac{e^{t}+1}{e^{t}-1}\right)-1}=t
$$

So the Equation (21) can be written

$$
\left|y_{\mu j}^{\prime}\right|=f\left(f\left(\left|2 \beta J_{\mu}\right|\right)+\sum_{l \in L(\mu) \backslash j} f\left(\left|x_{\mu l}^{\prime}\right|\right)\right)
$$
as

Using (10) and (11), the Equation (23) can be written

$$
\left|2 \beta y_{\mu j}\right|=f\left(f\left(\left|2 \beta J_{\mu}\right|\right)+\sum_{l \in L(\mu) \backslash j} f\left(\left|2 \beta x_{\mu l}\right|\right)\right)
$$

From (18) and (24), the check node processing in the statistical physics is denoted by 


$$
\begin{aligned}
& y_{\mu j}= \\
& \prod_{l \in L(\mu) \backslash j} \operatorname{sign}\left(J_{\mu} x_{\mu l}\right) \times \frac{1}{2 \beta} \times \\
& f\left(f\left(\left|2 \beta J_{\mu}\right|\right)+\sum_{l \in L(\mu) \backslash j} f\left(\left|2 \beta x_{\mu l}\right|\right)\right)
\end{aligned}
$$

\subsection{BP-Based with TAP Approach}

In this section, we try to develop the approximation of the BP algorithm: the BP-Based [6] algorithm, with the TAP approach. The key idea of this algorithm is that $\sum_{t} f(t) \leq f\left(\min _{t} t\right)$. The inequality is clear while depicting the function $f(t)$ on the Figure 1.

The Equation (24) is then approximated by

$$
\begin{aligned}
\left|2 \beta y_{\mu j}\right| & =f\left(f\left(\left|2 \beta J_{\mu}\right|\right)+\sum_{l \in L(\mu) \backslash j} f\left(\left|2 \beta x_{\mu l}\right|\right)\right) \\
& \approx f\left(f\left(\min _{l \in L(\mu) \backslash j}\left(\left|2 \beta J_{\mu}\right|,\left|2 \beta x_{\mu l}\right|\right)\right)\right) \\
& =\min _{l \in L(\mu) \backslash j}\left(\left|2 \beta J_{\mu}\right|,\left|2 \beta x_{\mu l}\right|\right)
\end{aligned}
$$

So, the extrinsic information in the BP-Based algorithm written with the TAP equation is given by:

$$
\begin{aligned}
y_{\mu j} & =\prod_{l \in L(\mu) \backslash j} \operatorname{sign}\left(J_{\mu} x_{\mu l}\right) \times \frac{1}{2 \beta} \times \min _{l \in L(\mu) \backslash j}\left(\left|2 \beta J_{\mu}\right|,\left|2 \beta x_{\mu l}\right|\right) \\
& =\prod_{l \in L(\mu) \backslash j} \operatorname{sign}\left(J_{\mu} x_{\mu l}\right) \times \min _{l \in L(\mu) \backslash j}\left(\left|J_{\mu}\right|,\left|x_{\mu l}\right|\right)
\end{aligned}
$$

Therefore, there is an important simplification in the BP-Based algorithm with TAP approach since the check node update is replaced by a selection of the minimum input value.

\section{3. $\lambda$-min Algorithm with TAP Approach}

In this section, we try to develop the $\lambda$-min algorithm [7] with the TAP approach. In the check node update of (25), the magnitude processing is run using the function defined by (20). While depicting this function on the Figure 1 , it is clear that $\sum_{t} f(t)$ can be approximated by the maximal values of $f(t)$ which is obtained for the minimal values of $|t|$.

So, like Guilloud, et al. [7] we propose to compute (24) with only the $\lambda$ bits that participate in check $\mu$ and which have the minimum magnitude.

$$
\left|2 \beta y_{\mu j}\right|=f\left(f\left(\left|2 \beta J_{\mu}\right|\right)+\sum_{l \in L_{\lambda}(\mu) \backslash j} f\left(\left|2 \beta x_{\mu l}\right|\right)\right)
$$

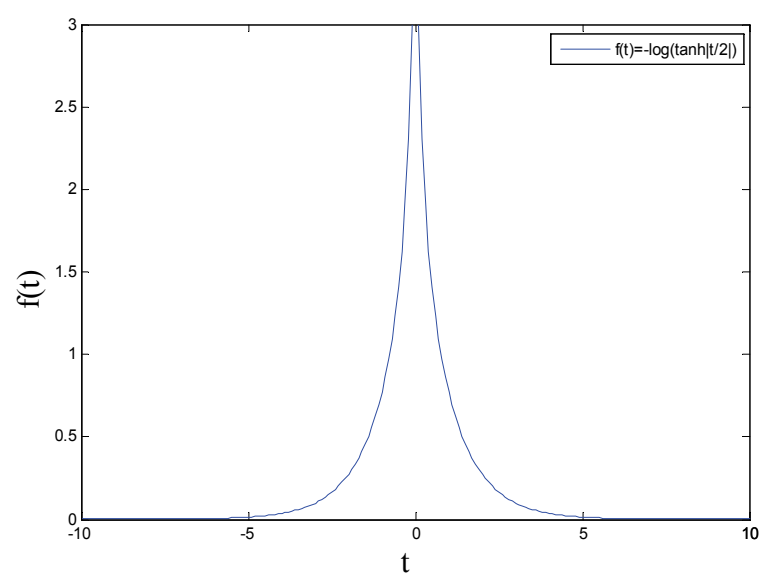

Figure 1. Shape of the function $f$.

With $L_{\lambda}(\mu)=\left\{j_{0}, \ldots, j_{\lambda-1}\right\}$ be the subset of $L(\mu)$ which contains the $\lambda$ bits that participate in check $\mu$ which $x_{\mu l}$ have the smallest magnitude.

The extrinsic information in the $\lambda$-min algorithm written with the TAP equation is given by (29)

$$
\begin{aligned}
& y_{\mu j}=\prod_{l \in L(\mu) \backslash j} \operatorname{sign}\left(J_{\mu} x_{\mu l}\right) \times \frac{1}{2 \beta} \times \\
& f\left(f\left(\left|2 \beta J_{\mu}\right|\right)+\sum_{l \in L_{\lambda}(\mu) \backslash j} f\left(\left|2 \beta x_{\mu l}\right|\right)\right)
\end{aligned}
$$

Two cases will occur: if the bit $j$ belongs to the subset $L_{\lambda}(\mu)$, then $y_{\mu j}$ are processed over $(\lambda-1)$ values of $L_{\lambda}(\mu) \backslash j$, otherwise $y_{\mu j}$ are processed over the $\lambda$ values of $L_{\lambda}(\mu)$. Hence, for the second case, the computations have to be performed only once [13].

\section{Simulation Results}

Simulations have been performed using regular $(3,6)$ LDPC code of length $N=1008$ and with 20 decoding iterations. We averaged the results over 10 codes. This ensemble of LDPC code is characterized by the same block length, the ones in each column and the ones in each row. For each run, a fixed code is used to generate 1008 bit codeword from 504 bit message. Corrupted versions of the codeword are then decoded using LLR-BP, BP-Based and $\lambda$-min decoding algorithms.

To observe the effect of the simplification in (27) and (29) on the performance from a statistical physics point of view, we have calculated the overlap between the actual message and the estimate (magnetization) for each fixed code and for each algorithm. After, we average the obtained results for the 10 different codes. Figure 2 depicts the magnetization performance of the LLR-BP, BP-Based and the $\lambda$-min algorithms. 
According the results of Figure 2, we can conclude the effectiveness of the $\lambda$-min algorithm. At a magnetization of 0.9 , the BP-Based algorithm introduces a degradation of $0.5 \mathrm{~dB}$ with 20 iterations. The 2-min algorithm introduces a degradation of $0.3 \mathrm{~dB}$. The performance of the 3-min algorithm is slightly worse than that of the LLR-BP algorithm, the degradation is only $0.08 \mathrm{~dB}$.

Another remark from Figure 2 is that at high signal to noise ratio the magnetization is concentrate at the value $m=1$. This value corresponds to the ferromagnetic state in the statistical physics and to the perfect decoding in the information theory.

The analogy between the Bit Error Rate (BER) performance and the magnetization performance can be examined in [14].

\section{Conclusions}

In this paper, we have been interested in the decoding of LDPC codes from a statistical physics approach. First, we have examined the correspondence between LDPC codes and Ising spin systems. The relation between the BP algorithm and TAP approach is investigated. Then, we showed that LLR-BP algorithm and its simplification i.e. the BP-Based algorithm and the $\lambda$-min algorithm can be obtained using the TAP approach of Ising spin systems in statistical physics. Besides, we have presented the performance of the decoding algorithms using a statistical physics parameter which is the magnetization.

Finally, we concluded that the BP-Based algorithm reduces the complexity for updating extrinsic information but there is degradation in performance compared to the LLR-BP algorithm. The $\lambda$-min algorithm reduces the complexity for updating extrinsic information without degradation in performance compared to the LLR-BP algorithm especially when $\lambda$ increases. These results con-

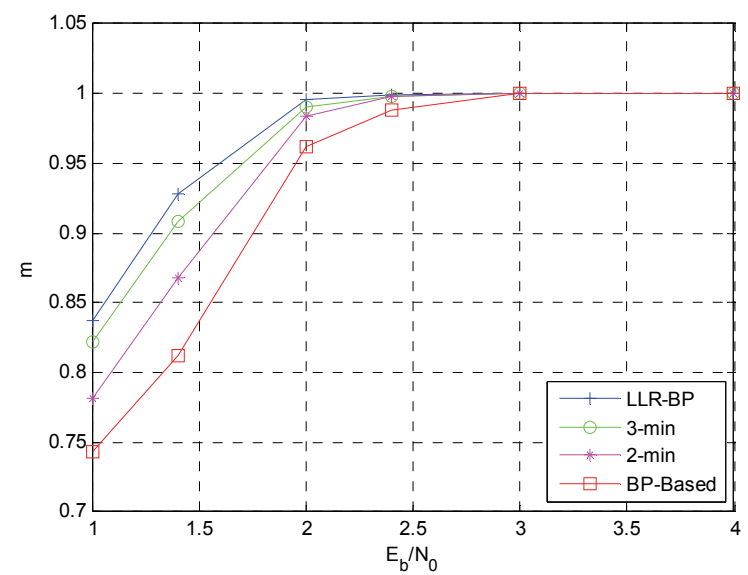

Figure 2. Magnetization performance of the LLR-BP, BPBased and $\lambda$-min algorithms with $\lambda=\{2,3\}$, for the $(1008,3$, 6) LDPC code ensemble and with 20 decoding iterations. firm with the results obtained in the information theory.

As a perspective of our work, we propose to study the replica method. This is a method from the statistical physics applied to find the magnetization. Besides, we propose to simplify the equations of this method by the same approximation in the BP-Based algorithm and the $\lambda$-min algorithm in order to compare the analytic results with the experimental ones.

\section{References}

[1] N. Sourlas, "Spin-Glass Models as Error-Correcting Codes," Nature, Vol. 339, No. 6227, 1989, pp.693-695.

[2] D. J. Thouless, P. W. Anderson and R. G. Palmer, "Solution of Solvable Model of A Spin Glass," Philosophical Magazine, Vol. 35, No. 3, 1977, pp. 593-601.

[3] R. G. Gallager, "Low-Density Parity-Check Codes," M.I.T. Press, Cambridge, Massachusetts, 1963.

[4] D. J. C. Mackay and R. M. Neal, "Near Shannon Limit Performance of Low Density Parity Check Codes," Electronics Letters, Vol. 32, No. 18, 1996, pp.1645-1646.

[5] D. J. C. Mackay, "Good Error-Correcting Codes Based on Very Sparse Matrices," IEEE Transactions on Information Theory, Vol. 45, No. 2, 1999, pp. 399-431.

[6] M. P. C. Fossorier, M. Mihaljevic and I. Imai, "Reduced Complexity Iterative Decoding of Low Density Parity Check Codes Based on Belief Propagation," IEEE Transactions on Communications, Vol. 47, No. 5, 1999, pp. 673-680.

[7] F. Guilloud, E. Boutillon and J. L. Danger, " $\lambda$-Min Decoding Algorithm of Regular and Irregular LDPC Codes," Proceedings of 3rd International Symposium on Turbo Codes \& Related Topics, Brest, 2003, pp. 451-454.

[8] Y. Kabashima and D. Saad, "Belief Propagation vs. TAP for Decoding Corrupted Messages," Europhysics Letters, Vol. 44, No. 5, 1998, pp. 668-674.

[9] T. Murayama, Y. Kabashima, D. Saad and R. Vicente, "Statistical Physics of Regular Low-Density Parity-Check Error-Correcting Codes," Physical Review E, Vol. 62, No. 2, 2000, pp. 1577-1591.

[10] R. Vicente, D. Saad and Y. Kabashima, "Finite-Connectivity Systems as Error-Correcting Codes," Physical Review E, Vol. 60, No. 5, 1999, pp. 5352-5366.

[11] J. Chen, A. Dholakia, E. Eleftheriou, M. Fossorier and X. Y. Hu, "Reduced-Complexity Decoding of LDPC Codes," IEEE Transactions on Communications, Vol. 53, No. 8, 2005, pp. 1288-1299.

[12] M. Mezard and A. Montanari, "Information, Physics and Computation," Oxford University Press, Oxford, 2008.

[13] F. Guilloud, "Generic Architecture for LDPC Codes Decoding," PhD Thesis, ENST Paris, 2004.

[14] M. Abdelhedi, O. Hamdi and A. Bouallegue, "Magnetization Performance of LDPC Decoding Algorithms," International Journal of Information and Coding Theory, 18 March 2010. 